\title{
A SEMI-IMPLICIT SCHEME FOR SOLVING INCOMPRESSIBLE VISCOUS FREE SURFACE FLOWS
}

C. M. Oishía , J. A. Cuminato ${ }^{\text {, }}$

V. G. Ferreira ${ }^{a}$, M. F. Toméa,

A. Castelo ${ }^{\mathrm{a}}$; and N. Mangiavacchi ${ }^{\mathrm{b}}$

${ }^{a}$ Instituto de Ciências Matemáticas e de Computação, ICMC-USP, C.P. 668, 13560-970,

São Carlos, SP, Brasil

\{oishi,jacumina,pvgf,murilo,castelo\}@

lcad.icmc.usp.br

bDepartamento de Engenharia Mecânica, UERJ,

Rua São Francisco Xavier, 524, 20550-900, Rio

de Janeiro, RJ, Brasil

norberto@uerj.br

\section{ABSTRACT}

The present work is concerned with a numerical method for solving the two-dimensional time-dependent incompressible Navier-Stokes equations in the primitive variables formulation. The diffusive terms are treated by Implicit Backward and Crank-Nicolson methods, and the non-linear convection terms are, explicitly, approximated by the high order upwind VONOS (Variable-Order Non-Oscillatory Scheme) scheme. The boundary conditions for the pressure field at the free surface are treated implicitly, and for the velocity field explicitly. The numerical method is then applied to the simulation of free surface and confined flows. The numerical results show that the present technique eliminates the stability restriction in the original explicit method. For low Reynolds number flow dynamics, the method is robust and produces numerical results that compare very well with the analytical solutions.

Keywords: Navier-Stokes equations; Finite-Difference method; Semi-Implicit method.

\section{NOMENCLATURE}

$\begin{array}{ll}F r & \text { Froude number } \\ G & \text { gravity field, } \mathrm{m} / \mathrm{s}^{2} \\ \boldsymbol{I} & \text { identity tensor } \\ L & \text { length scale, } \mathrm{m} \\ p & \text { dimensionless pressure } \\ R e & \text { Reynolds number } \\ \boldsymbol{T} & \text { total tensor } \\ t & \text { dimensionless time } \\ U & \text { velocity scale, } \mathrm{m} / \mathrm{s} \\ u, v & \text { dimensionless velocity components } \\ x, y & \text { dimensionless cartesian coordinates }\end{array}$

\section{Greek symbols}

$\delta t \quad$ dimensionless time-step

$\delta x, \delta y$ dimensionless grid spacing

$v \quad$ fluid kinematic viscosity, $\mathrm{m}^{2} / \mathrm{s}$

$\tau \quad$ stress tensor

\section{INTRODUCTION}

In many fluid flow problems, the viscous forces are dominant, and several numerical techniques have been developed for the solution of this class of flows. In these fluid flow problems, the Reynolds number is often much smaller than 1. Due to this fact, numerical techniques that apply an explicit formulation, as the GENSMAC (GENeralized Simplified Marker-And-Cell) method (Tomé and Mckee, 1994), introduce the parabolic stability restriction, making the time step very small for some applications, justifying the need for methods with better stability properties.

In the present paper, a semi-implicit finite difference numerical method for solving incompressible viscous free surface fluid flow problems is outlined. By using implicit formulation, this method eliminates the stability restrictions in the explicit formulation. Therefore, it is proposed a modification in the GENSMAC method adding implicit schemes and treating the boundary conditions for the pressure field at the free surface implicitly.

As GENSMAC methodology, the produced time-marching is based on projection methods (Chorin, 1967; Gresho, 1990). It is a finite difference technique based on a staggered grid that solves the full Navier-Stokes equations in primitive variables. In particular, it solves problems with free surfaces.

In non-dimensional conservative form, the equations for incompressible viscous Newtonian flows are

$$
\begin{gathered}
\frac{\partial \boldsymbol{u}}{\partial t}+\nabla \cdot(\boldsymbol{u} \boldsymbol{u})=-\nabla p+\frac{1}{\operatorname{Re}} \nabla^{2} \boldsymbol{u}+\frac{1}{F r^{2}}, \\
\nabla \cdot \boldsymbol{u}=0,
\end{gathered}
$$

where $t$ is time, $\boldsymbol{u}=[u(x, y, t), v(x, y, t)]$ is the velocity field, $p=p(x, y, t)$ is pressure per unit of 
mass and $\boldsymbol{g}=\left(g_{x}, g_{y}\right)$ is the gravity field. The nondimensional parameters $R e=\frac{L U}{v}$ and $F r=\frac{U}{\sqrt{g L}}$ are the Reynolds and Froude numbers, respectively, being $L$ and $U$ the length and the velocity scales, and $v$ the kinematic viscosity coefficient of the fluid.

\section{NUMERICAL METHOD}

The numerical method proposed to solve Eqs. (1a) and (1b) is basically a modification of the GENSMAC method. Firstly, a provisional velocity field $\tilde{\boldsymbol{u}}$ is calculated from Eq. (1a), that is,

$$
\frac{\partial \tilde{\boldsymbol{u}}}{\partial t}+\nabla \cdot(\boldsymbol{u} \boldsymbol{u})=-\nabla \tilde{p}+\frac{1}{R e} \nabla^{2} \boldsymbol{u}+\frac{1}{F r^{2}}
$$

where $\tilde{p}$ is a provisional pressure. Generally, this provisional velocity field is not a solenoidal field, so $\tilde{p} \neq p$. For $t=t_{0}$, it is considered that $\boldsymbol{u}\left(\boldsymbol{x}, t_{0}\right)$ and $\tilde{\boldsymbol{u}}\left(\boldsymbol{x}, t_{0}\right)$ satisfy the same boundary conditions and that on the boundary $\boldsymbol{u}\left(\boldsymbol{x}, t_{0}\right)=\tilde{\boldsymbol{u}}\left(\boldsymbol{x}, t_{0}\right)$. The main modifications in the GENSMAC method were the inclusion of the Implicit Formulations (IF) for two variations in the projection methods.

The first projection method is based on the solution of the time-discretized Eq. (1a), without a provisional pressure gradient (referred here as pressure-free projection method, (Chorin, 1967) and denoted by $P 1$ ). Other modification in this equation is the application of implicit methods for the viscous terms. The implicit schemes used in $P 1$ were the Backward Implicit $(B I)$ and CrankNicolson $(C N)$ methods. In order to improve the temporal accuracy, a 2-step Adams method was employed. This method uses the $C N$ approximation for the viscous terms and the explicit AdamsBashforth for the non-linear convective terms of Eq. (2). This method is known as Adams-Bashforth/ Crank-Nicolson $(A B / C N)$. Therefore, applying the method $P 1$ and using the implicit formulations, Eq. (2) is rewritten in the following way:

- P1-BI method

$$
\tilde{\boldsymbol{u}}-\frac{\delta t}{R e} \nabla^{2} \tilde{\boldsymbol{u}}=\boldsymbol{u}^{n}+\delta t\left(-\nabla \cdot(\boldsymbol{u} \boldsymbol{u})^{n}+\frac{1}{F r^{2}}\right.
$$

- P1-CN method

$$
\tilde{\boldsymbol{u}}-\frac{\delta t}{2 \operatorname{Re}} \nabla^{2} \tilde{\boldsymbol{u}}=\boldsymbol{u}^{n}+\delta t\left(-\nabla \cdot(\boldsymbol{u} \boldsymbol{u})^{n}+\frac{1}{2 R e} \nabla^{2} \boldsymbol{u}^{n}+\frac{1}{F r^{2}} \boldsymbol{g}^{n}\right)
$$

\section{- $\quad P 1-A B / C N$ method}

$$
\begin{aligned}
& \tilde{\boldsymbol{u}}-\frac{\delta t}{2 \operatorname{Re}} \nabla^{2} \widetilde{\boldsymbol{u}}=\boldsymbol{u}^{n}+\delta t\left(-\frac{3}{2} \nabla \cdot(\boldsymbol{u} \boldsymbol{u})^{n}+\frac{1}{2} \nabla \cdot(\boldsymbol{u} \boldsymbol{u})^{n-1}+\frac{1}{2 \operatorname{Re}} \nabla^{2} \boldsymbol{u}^{n}\right. \\
& \left.+\frac{1}{F r^{2}} \boldsymbol{g}^{n}\right)
\end{aligned}
$$

Using the theory of the projection methods, a general velocity field can be decomposed into a tentative $\tilde{\boldsymbol{u}}$ and the gradient of a potential $\nabla \psi$. In the $P 1$ method, the function $\psi$ is calculated in the whole domain. The second projection method used in this work is based on the method with the provisional pressure gradient as in Eq. (2), referred to incremental-pressure projection methods, and denoted here by $P 2$ (Gresho, 1990). In the same manner as in the method of $P 1$, the viscous terms were taken implicitly, and Eq. (2) for $P 2$ becomes:

- P2-BI method

$$
\tilde{\boldsymbol{u}}-\frac{\delta t}{R e} \nabla^{2} \tilde{\boldsymbol{u}}=\boldsymbol{u}^{n}+\delta t\left(-\nabla \cdot(\boldsymbol{u} \boldsymbol{u})^{n}-\nabla \tilde{p}\right.
$$

- P2-CN method

$$
\begin{aligned}
& \widetilde{\boldsymbol{u}}-\frac{\delta t}{2 R e} \nabla^{2} \widetilde{\boldsymbol{u}}=\widetilde{\boldsymbol{u}}^{n}+\delta t\left(-\nabla \cdot(\boldsymbol{u} \boldsymbol{u})^{n}-\nabla \widetilde{p}+\frac{1}{2 R e} \nabla^{2} \boldsymbol{u}^{n}\right. \\
& \left.+\frac{1}{F r^{2}} \boldsymbol{g}^{n}\right)
\end{aligned}
$$

\section{- $P 2-A B / C N$ method}

$$
\begin{aligned}
& \widetilde{\boldsymbol{u}}-\frac{\delta t}{2 R e} \nabla^{2} \widetilde{\boldsymbol{u}}=\boldsymbol{u}^{n}+\delta t\left(-\frac{3}{2} \nabla \cdot(\boldsymbol{u} \boldsymbol{u})^{n}+\frac{1}{2} \nabla \cdot(\boldsymbol{u} \boldsymbol{u})^{n-1}\right. \\
& \left.-\nabla \widetilde{p}+\frac{1}{2 \operatorname{Re}} \nabla^{2} \boldsymbol{u}^{n}+\frac{1}{F r^{2}} \boldsymbol{g}^{n}\right)
\end{aligned}
$$

The development of the $P 2$ method using $I F$ is similar to the $P 1$ method, with the difference that now $\tilde{p} \neq 0$ will be calculated. In the GENSMAC method, the Poisson equation for $\psi$ is applied for the whole domain containing fluid, with the appropriate boundary conditions described in 
(Tomé and McKee, 1994). For the P1 and P2 methods using $I F$, besides the Poisson equation, a new equation is imposed on the potential $\psi$ for the fluid free surface. This new equation is calculated from the equation of the pressure in the free surface. On the free surface, it is necessary to impose conditions on the velocity and pressure. These conditions, considering absent surface tension, are summarized as

$$
(\boldsymbol{T} . \boldsymbol{n}) . \boldsymbol{n}=0 \quad \text { and } \quad(\boldsymbol{T} . \boldsymbol{n}) . \boldsymbol{m}=0,
$$

where $\boldsymbol{n}=\left(n_{x}, n_{y}\right)$ is the normal vector, external to the surface, and $\boldsymbol{m}=\left(m_{x}, m_{y}\right)$ is the tangent vector to the free surface. Substituting the total tensor $\boldsymbol{T}=-p \boldsymbol{I}+\tau$, where $\tau$ is the stress tensor and $\boldsymbol{I}$ the identity tensor, in Eq. (5) it may be found

$$
\begin{gathered}
-p+\frac{2}{\operatorname{Re}}\left(\frac{\partial u}{\partial x} n_{x}^{2}+\frac{\partial v}{\partial y} n_{y}^{2}+\left(\frac{\partial u}{\partial y}+\frac{\partial v}{\partial x}\right) n_{x} n_{y}\right)=0 \\
2 \frac{\partial u}{\partial x} n_{x} m_{x}+2 \frac{\partial v}{\partial y} n_{y} m_{y}+\left(\frac{\partial u}{\partial y}+\frac{\partial v}{\partial x}\right)\left(n_{y} m_{x}+n_{x} m_{y}\right)=0
\end{gathered}
$$

The equations for $\psi$ at the free surface are derived from the equation of the pressure Eq. (6) with implicit velocity, that is, the boundary conditions on the free surfaces are also taken implicitly. The application of $P 1$ and $P 2$ methods for the implicit formulations in GENSMAC results in 3 sparse linear systems: 2 due to the equations that calculate the intermediary velocity and 1 due to the calculation of the potential $\psi$. When the implicit formulations are applied, for the $B I$ or $C N$ methods, the viscous terms are taken implicitly, and for that, it is necessary to solve systems for velocities $\tilde{u}$ and $\tilde{v}$. The linear systems resulting from Eqs. (3a)-(4c) are sparse, positive defined and symmetric. Due to those properties, an efficient method is the Conjugated Gradient (CG) method. The linear system for $\psi$ is sparse, but non-symmetric, and therefore the method used was the Bi-Conjugated Gradients with Preconditioning (BCGP). Besides the method BCGP, there are other alternatives recommended in the literature for sparse problems such as: the Generalized Minimum Residual (GMRES) and Preconditioned Conjugate Gradient Squared
(PCGS). More details of the numerical methods using implicit formulations and boundary conditions at the free surfaces can be found in Oishi (2004).

\section{STABILITY OF P1 AND P2 METHODS}

The stability restriction imposed for explicit treatment of the viscous terms requires that:

$$
\delta t_{\text {viscous }} \leq 0.5 \operatorname{Re}\left[(\delta x)^{-2}+(\delta y)^{-2}\right]^{-1}
$$

where $\delta x$ and $\delta y$ are the grid spacing. The application of Implicit Formulations for the viscous terms as in Eqs. (3a)-(4c), can, in principle, remove the restriction shown in Eq. (8). Therefore, the restrictions on $\delta t$ for $P 1$ and $P 2$ using $I F$ are more relaxed than in the original GENSMAC code.

\section{SOLUTION PROCEDURE}

The sequence of steps in the solution procedure purports updating the discreet variables, starting from an initial time $t_{n}$. The algorithm is described as follows:

Step 1: For the $P 1$ method, the pressure gradient $\nabla \tilde{p}$ is eliminated from the formulation and the velocity at the free surfaces is calculate from Eq. (7). For the $P 2$ method, besides the calculation of the velocity at the free surfaces, the pressure gradient is conserved, $\tilde{p}=p^{n}$, where $p^{n}$ is the pressure calculated in the previous time from Eq. (6);

Step 2: Calculate an intermediary velocity field $\tilde{\boldsymbol{u}}(\boldsymbol{x}, t)$ in $t=t_{n}+\delta t$. When the $P 1$ method is used, Eqs. (3a)-(3c) can be used. Similarly, when the $P 2$ method is applied, Eqs. (4a) - (4c) can be used;

Step 3: Solve the Poisson equation for the potential $\psi$ in the regions that contain fluid, and at the free surfaces, calculate $\psi$ from Eq. (6) with implicit velocity. Details of the boundary conditions for the Poisson equation and the equations for $\psi$ at the free surfaces can be found in Oishi (2004);

Step 4: Compute the corrected velocity field

$$
\boldsymbol{u}^{n+1}=\tilde{\boldsymbol{u}}-\nabla \psi^{n+1}
$$


Step 5: Compute the final pressure field. For the $P 1$ method, the pressure is computed from equation

$$
p^{n+1}=\frac{\psi^{n+1}}{\delta t},
$$

and for the $P 2$ method, the equation is

$$
p^{n+1}=\tilde{p}+\frac{\psi^{n+1}}{\delta t}
$$

Step 6: Update the marker particles positions. The last step in the calculation is moving the marker particles to their new positions. This is performed by solving

$$
\frac{d x}{d t}=u \text { and } \frac{d y}{d t}=v
$$

by Euler's method. The fluid surface is defined by a list containing these particles and the visualization of this boundary is obtained by connecting them by straight lines.

\section{DISCRETIZATION}

Equations (1a) and (1b) are approximated in a staggered mesh. In this mesh, the pressure is stored at cell centers and the components of the velocity $u$ and $v$ are stored in the middle of the lateral faces. As in Tomé and McKee (1994), the diffusion terms and the pressure gradient in Eqs. (3a)-(4c) are approximated by central differences, whereas the time derivatives are approximated by forward differences (Euler explicit). The convective terms are discretized by the VONOS scheme (see Ferreira et al., 2002), which is a bounded upwind technique. For solving the conservation equations, the FREEFLOW2D (see Castelo et al., 2000) simulation environment is used.

This systems is composed of three module: a modeling module (modeler) a simulation module (simulator, which implements the full Navier-Stokes equations and mass conservation equation) and the visualization module (visualizator).

\section{NUMERICAL RESULTS}

In this section, numerical results using the implicit formulations are presented. The main aim in the comparison is assessing the efficiency of $P 1$ and $P 2$ methods using $B I, C N$ and $A B / C N$ formulations, in relation to the explicit method for problems with $R e<1$. The results are encouraging, in terms of accuracy and efficiency. The following test cases are considered.

\section{Hagen-Poiseuille Flow}

The validation of the numerical results using $P 1$ and $P 2$ methods with $I F$ was performed on the flow of a fluid between two parallel plates. In this test case, comparisons between the numerical solutions and the analytical solution are feasible (see Batchelor, 1970). In this simulation, two parallel plates separated by a distance $L=1 \mathrm{~m}$ are considered, forming a channel, that is initially empty and through which the fluid is injected in its entrance region with parabolic velocity profile. The $P 1$ and $P 2$ methods using $I F$ were applied using three meshes, defined respectively as coarse (M1, where $\delta x=\delta y=0.1)$; middle (M2, where $\delta x=\delta y=0.05)$, and fine (M3, where $\delta x=\delta y=0.025)$ meshes. In order to show the convergence of the methods presented in this work the relative error, in the $l_{2}$ norm, between the numerical solutions and the analytic was calculated. These results are presented in Tab. 1.

It can be observed from Tab. 1 that the numerical results are similar to the analytic solution, that is, the numerical values obtained by the $P 1$ and $P 2$ methods, on the three meshes, are in good agreement with the analytic solution. For creep flow problems, the $I F$ was more stable than the original explicit method. Table 2 shows the $\delta t$ allowed by implicit and explicit formulations. The methods that use the formulation $B I$ admitted values of $\delta t$ larger than the other formulations. When Re decreases, the restriction on the time step for the explicit method (Eq. 8 ) was overcome by the $P 1$ and $P 2$ methods. From Tab. 2, it can be seen that the methods using the formulation $B I$ demanded a $\delta t$ about 500 to 500,000 times larger than the explicit method, when Re decreases, whereas the formulations $C N$ and $A B / C N$ presented $\delta t$ about 20 times larger, regardless of the Re. 
Table 1. Results of Hagen-Poiseuille flow for $R e=0.1$ in the meshes M1, M2 and M3

\begin{tabular}{|l|c|c|}
\hline \multicolumn{1}{|c|}{ Method } & \multicolumn{1}{c|}{$\boldsymbol{t}$} & M1 \\
\hline Explicit & $1.0 \times 10^{-4}$ & $2.5 \times 10^{-5}$ \\
\hline$P 1-B I$ & $1.25 \times 10^{-3}$ & $7.1 \times 10^{-4}$ \\
\hline$P 1-C N$ & $2.0 \times 10^{-3}$ & $5.6 \times 10^{-4}$ \\
\hline$P 1-A B / C N$ & $2.0 \times 10^{-3}$ & $1.6 \times 10^{-4}$ \\
\hline$P 2-B I$ & $1.0 \times 10^{-2}$ & $2.5 \times 10^{-5}$ \\
\hline$P 2-C N$ & $2.0 \times 10^{-3}$ & $2.5 \times 10^{-5}$ \\
\hline$P 2-A B / C N$ & $2.0 \times 10^{-3}$ & $2.5 \times 10^{-5}$ \\
\hline \multicolumn{1}{|c|}{ Method } & $\delta \boldsymbol{t}$ & $\mathbf{M} 2$ \\
\hline Explicit & $2.5 \times 10^{-5}$ & $1.8 \times 10^{-6}$ \\
\hline$P 1-B I$ & $2.5 \times 10^{-4}$ & $3.7 \times 10^{-5}$ \\
\hline$P 1-C N$ & $5.0 \times 10^{-4}$ & $4.5 \times 10^{-5}$ \\
\hline$P 1-A B / C N$ & $5.0 \times 10^{-4}$ & $2.5 \times 10^{-5}$ \\
\hline$P 2-B I$ & $1.25 \times 10^{-2}$ & $1.8 \times 10^{-6}$ \\
\hline$P 2-C N$ & $5.0 \times 10^{-4}$ & $1.8 \times 10^{-6}$ \\
\hline$P 2-A B / C N$ & $5.0 \times 10^{-4}$ & $1.8 \times 10^{-6}$ \\
\hline \multicolumn{1}{|c|}{ Method } & $\delta \boldsymbol{t}$ & $\mathbf{M 3}$ \\
\hline Explicit & $6.25 \times 10^{-6}$ & $1.3 \times 10^{-7}$ \\
\hline$P 1-B I$ & $6.25 \times 10^{-5}$ & $3.2 \times 10^{-6}$ \\
\hline$P 1-C N$ & $1.25 \times 10^{-4}$ & $3.2 \times 10^{-6}$ \\
\hline$P 1-A B / C N$ & $1.25 \times 10^{-4}$ & $2.1 \times 10^{-6}$ \\
\hline$P 2-B I$ & $6.25 \times 10^{-3}$ & $1.2 \times 10^{-7}$ \\
\hline$P 2-C N$ & $5.0 \times 10^{-4}$ & $1.1 \times 10^{-7}$ \\
\hline$P 2-A B / C N$ & $5.0 \times 10^{-4}$ & $1.1 \times 10^{-7}$ \\
\hline
\end{tabular}

\section{Simulation of Container Filling}

In this test case, the filling of a container with a Newtonian fluid with $R e=0.1$ is considered. In this simulation a comparison of CPU time was made using the $P 1$ and $P 2$ methods with $I F$, and the explicit method. For these models, a mesh $\delta x=\delta y=0.0005$ was used for all methods. The gravitational field acts on the flow and the final time of the simulations was $t=100$. An illustration is presented in Fig. 1 where the behavior of the flow can be observed.

The results obtained by the $P 1$ method, using the $B I, C N$ and $A B / C N$ formulations and those by $P 2$ method using the $B I$ and $A B / C N$ formulations were very similar to those of the $P 2$ method using the $C N$ formulation. In Fig. 1 one of the results is presented. A comparison between the methods that use the implicit and explicit formulations, regarding the value of $\delta t$ allowed for each method, the number of iterations and the CPU time for the time $t=5.6$, can be seen in Tab. 3. Again, the implicit formulations overcame the restriction of stability of the original explicit method. These methods used less iterations to obtain the solution at the time $t=5.6$.
Table 2. Limited of stability for $\delta t$ in HagenPoiseuille flow over the mesh M2, with different Re values

\begin{tabular}{|l|c|c|c|c|}
\hline Method & $\mathrm{Re}=10^{-1}$ & $\mathrm{Re}=10^{-2}$ & $\mathrm{Re}=10^{-3}$ & $\mathrm{Re}=10^{-4}$ \\
\hline Explicit & $2.5 \times 10^{-5}$ & $2.5 \times 10^{-6}$ & $2.5 \times 10^{-7}$ & $2.5 \times 10^{-8}$ \\
\hline$P 1-B I$ & $1.25 \times 10^{-2}$ & $1.25 \times 10^{-2}$ & $1.25 \times 10^{-2}$ & $1.25 \times 10^{-2}$ \\
\hline$P 1-C N$ & $5.0 \times 10^{-4}$ & $5.0 \times 10^{-5}$ & $5.0 \times 10^{-6}$ & $5.0 \times 10^{-7}$ \\
\hline$P 1-A B / C N$ & $5.0 \times 10^{-4}$ & $5.0 \times 10^{-5}$ & $5.0 \times 10^{-6}$ & $5.0 \times 10^{-7}$ \\
\hline$P 2-B I$ & $1.25 \times 10^{-2}$ & $1.25 \times 10^{-2}$ & $1.25 \times 10^{-2}$ & $1.25 \times 10^{-2}$ \\
\hline$P 2-C N$ & $5.0 \times 10^{-4}$ & $5.0 \times 10^{-5}$ & $5.0 \times 10^{-6}$ & $5.0 \times 10^{-7}$ \\
\hline$P 2-A B / C N$ & $5.0 \times 10^{-5}$ & $5.0 \times 10^{-5}$ & $5.0 \times 10^{-6}$ & $5.0 \times 10^{-7}$ \\
\hline
\end{tabular}

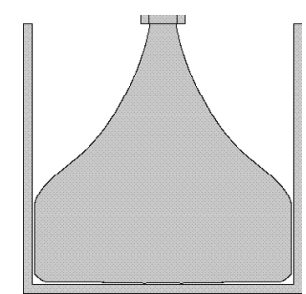

Figure 1. Numerical simulation of container filling, with $R e=0.1$ and simulation time $t=5.6$, for the $P 2$ method using the $C N$ formulation

Table 3. Results for simulation of container filling. Input data employed: $L=0.05 \mathrm{~m}, U=1.0 \mathrm{~m} / \mathrm{s}$, and $t=5.6$

\begin{tabular}{|l|c|c|c|}
\hline Method & $\delta t$ & $\begin{array}{c}\text { Number of } \\
\text { iteration }\end{array}$ & $\begin{array}{c}\text { CPU time- } \\
\text { minutes:seconds }\end{array}$ \\
\hline Explicit & $5.0 \times 10^{-7}$ & 559998 & $430: 59$ \\
\hline$P 1-B I$ & $3.0 \times 10^{-5}$ & 11200 & $41: 52$ \\
\hline$P 1-C N$ & $1.0 \times 10^{-5}$ & 28000 & $99: 16$ \\
\hline$P 1-A B / C N$ & $1.0 \times 10^{-5}$ & 28000 & $106: 18$ \\
\hline$P 2-B I$ & $6.0 \times 10^{-5}$ & 8960 & $21: 41$ \\
\hline$P 2-C N$ & $1.0 \times 10^{-5}$ & 28000 & $92: 51$ \\
\hline$P 2-A B / C N$ & $1.0 \times 10^{-5}$ & 28000 & $96: 25$ \\
\hline
\end{tabular}

\section{Comparison with Experimental Results}

Finally, in this test case, qualitative comparisons between numerical results with the experiments described by (Tomé et al., 1999) are carried out. For this model, a mesh of $\delta x=\delta y=0.0005$ was used for all methods, with the gravitational field acting on the flow and a final time of $t=100$. Figure 2 presents the comparison between the numerical solution and an experimental configuration. In this figure, the numerical method used was the $P 2$ method with the $B I$ formulation. The other methods that use the implicit formulations are not displayed because they showed similar results to the $P 2$ method using the formulation $B I$. The results obtained with the explicit method (Tomé et al., 1999) were very close to those of the implicit formulations. However, the 
time-step of the explicit method became very small due to the parabolic stability restriction (Eq. 8). The implicit formulations, as previously presented, allowed larger values for $\delta t$, overcoming the restriction of the original explicit method.

Therefore, the main difference between this work and that of Tomé et al. (1999) was the significant CPU cost saving. This fact is very important in engineering applications.

a)

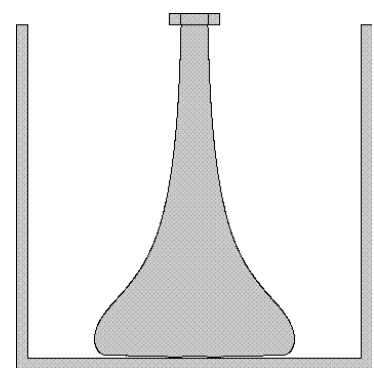

b)

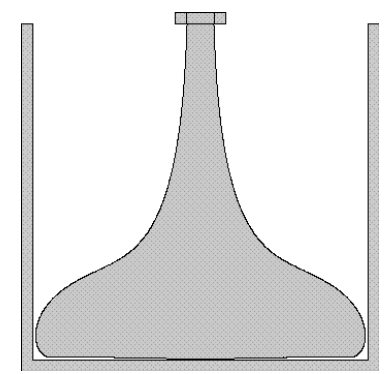

c)

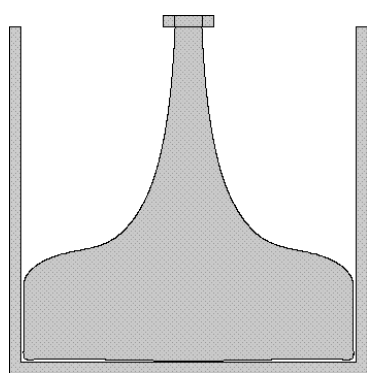

d)

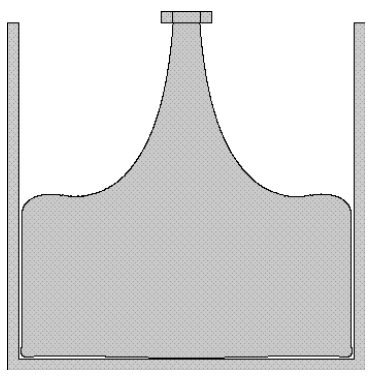

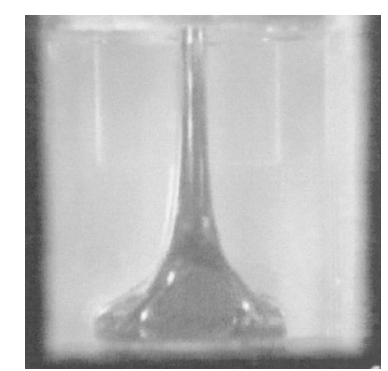
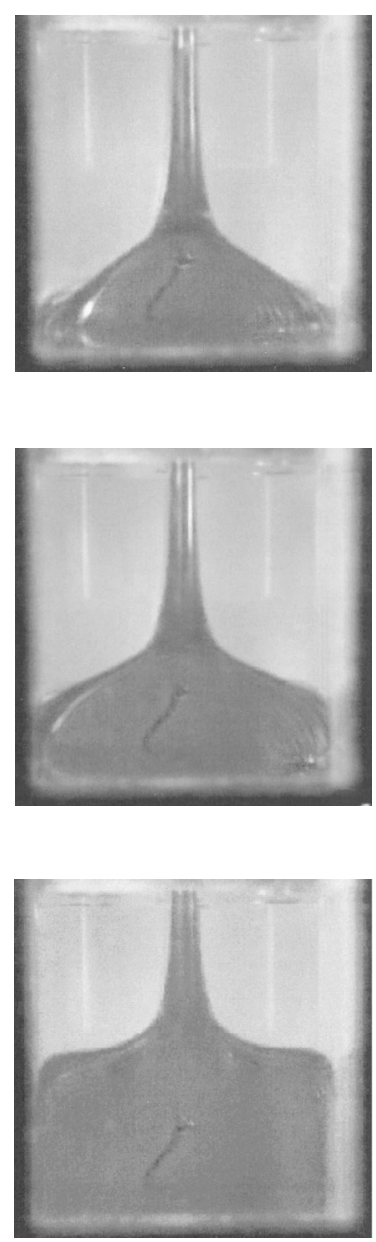

Figure 2. Experimental solution (left) and numerical (right) solution by using $P 2$ method with the $B I$ formulation. a) $t=35$, b) $t=55$, c) $t=65$ and d) $t=85$. The fluid used in this simulation was the glucose syrup solution (see Tomé et al., 1999)

\section{CONCLUSIONS}

The main purpose of this work is the design and analysis of implicit numerical schemes, which can be used in conjunction with the GENSMAC method, for the simulation of transient viscous incompressible Newtonian flows with free surface.

A modification was made to the implicit treatment of boundary conditions for pressure at the free surface. The implicit formulations presented satisfactory results for unsteady free surface flows. The validation showed the comparison between the analytic solution and the numerical solution of the $P 1$ and $P 2$ methods using $I F$. The numerical results show the capacity of these semi-implicit methods of simulating fluid flow with free surface. However, the $C N$ and $A B / C N$ formulations introduced numerical oscillations, and as a consequence, the allowed value of $\delta t$ was more restrict than that of the $B I$ formulation. More details about the numerical oscillations of the method $C N$ can be found in Hirsch (1989) and Turek (1996).

Although the $C N$ and $A B / C N$ formulations have allowed a time step larger than that of the original explicit method, the $B I$ formulation proved to be stable allowing very large values of $\delta t$. Care is recommended in choosing the time step so that numerical accuracy is not affected. In all the simulations, the implicit formulations overcame the value of the time step of the explicit method and, in some cases, the $\delta t$ was approximately 500000 times larger than the one of the explicit method.

The $P 1$ and $P 2$ methods using the implicit formulations presented similar errors to those of the explicit method with a much smaller number of iterations. The processing time demanded by the implicit formulations was significantly shorter than that of the explicit formulation. Therefore, the $P 1$ and $P 2$ methods using the implicit formulations showed to be capable of solving viscous problems with free surfaces.

\section{ACKNOWLEDGEMENTS}

This work was financially supported by FAPESP, contracts 03/12612-9 and 00/03385-0.

\section{REFERENCES}

Batchelor, G. K., 1970, An Introduction to Fluid Dynamics, Cambridge. 
Castelo, A., Tomé, M. F., César, M., Cuminato, J. A., and McKee, S., 2000, Freeflow: An Integrated Simulation System for ThreeDimensional Free Surface Flows, Journal of Computers and Visualization in Science, Vol. 2, pp 199-210.

Chorin, A., 1967, A Numerical Method for Solving Incompressible Viscous Flow Problems, Journal of Computational Physics, Vol. 2, pp. 12-26.

Ferreira, V. G., Tomé, M. F., Castelo, A., Cuminato, J. A., Mangiavacchi, N., and McKee, S., 2002, High Order Upwinding and the Hydraulic Jump, International Journal for Numerical Methods in Fluids, Vol. 39, No. 7, pp. 549-583.

Gresho, P. M., 1990, On the Theory of Semi-Implicit Projection Methods for Viscous Incompressible Flow and its Implementation via a Finite Element Method that also Introduces a Nearly Consistent Mass Matrix, International Journal for Numerical Methods in Fluids, Vol. 11, No. 5, pp. 587-620.

Hirsch, C., 1989, Numerical Computational of Internal and External Flows, Vol. 1, A Wiley-Interscience Publication.

Oishi, C. M., 2004, Análise e Implementação de Métodos Implícitos no Sistema FREEFLOW2D, Master's Dissertation, University of São Paulo, Brazil. (in Portuguese)

Tomé, M. F. and McKee, S., 1994, GENSMAC: A Computational Marker-And-Cell Method for Free Surface Flows in General Domains, Journal of Computational Physics, Vol. 110, No. 1, pp. 171-186

Tomé, M. F., McKee, S., Barrant, L., Jarvis, D., and Patrick, A., 1999, An Experimental and Numerical Investigation of Container Filling with Viscous Liquids, International Journal for Numerical Methods in Fluids, Vol. 31, No. 8, pp. 1333-1353.

Turek, S., 1996, A Comparative Study of Some Time-Stepping Techniques for the Incompressible Navier-Stokes Equations: from Fully Implicit Nonlinear Schemes to SemiImplicit Projection Methods, International Journal for Numerical Methods in Fluids, Vol. 22, No. 10, pp. 987-1011. 